\title{
UNIQUENESS AND DECAY PROPERTIES OF MARKOV BRANCHING PROCESSES WITH DISASTERS
}

\author{
ANYUE CHEN, ${ }^{*}$ South University of Science and Technology of China \\ and University of Liverpool \\ KAI WANG NG, ${ }^{* *}$ University of Hong Kong \\ HANJUN ZHANG, ${ }^{* * *}$ Xiangtan University
}

\begin{abstract}
In this paper we discuss the decay properties of Markov branching processes with disasters, including the decay parameter, invariant measures, and quasistationary distributions. After showing that the corresponding $q$-matrix $Q$ is always regular and, thus, that the Feller minimal $Q$-process is honest, we obtain the exact value of the decay parameter $\lambda_{C}$. We show that the decay parameter can be easily expressed explicitly. We further show that the Markov branching process with disaster is always $\lambda_{C}$-positive. The invariant vectors, the invariant measures, and the quasidistributions are given explicitly.
\end{abstract}

Keywords: Markov branching process; disaster; decay parameter; invariant measure; invariant vector; quasistationary distribution

2010 Mathematics Subject Classification: Primary 60J27

Secondary $60 \mathrm{~J} 35 ; 60 \mathrm{~J} 80$

\section{Introduction}

Markov branching processes (MBPs) with or without disasters occupy a major niche in the theory and applications of probability theory. Good references for the latter are, among many others, [2] and [10]. Although the number of references in the literature for MBPs with disasters is far less than that for MBPs without disasters, the importance, both in theory and application, of MBPs with disasters is well recognised. This is in particular because the existence of disasters is well known to significantly change the behavior of the branching process. Also, from the point of view of applications, the effect of disasters is extremely important. Kaplan et al. [12], for example, discussed the effect of disasters on the extinction probabilities of branching processes. A special kind of disaster in which the whole population instantly goes extinct, usually referred to as killing, has attracted particular attention due to its fatal and catastrophic effect on the whole population. For example, as early as 1982, Karlin and McGreger [13] thoroughly analyzed the properties of the birth-death process with killing. In this paper we primarily discuss the properties of MBPs with killing, but we still use the terminology MBPs with disasters.

\footnotetext{
Received 20 September 2012; revision received 2 August 2013.

* Postal address: Department of Financial Mathematics and Financial Engineering, South University of Science and Technology of China, Shenzhen, Guangdong, 518055, P. R. China. Email address: chen.ay@ sustc.edu.cn

** Postal address: Department of Statistics and Actuarial Science, University of Hong Kong, Pokfulam Road, Hong Kong. Email address: kaing@hku.hk

*** Postal address: School of Mathematics and Computational Science, Xiangtan University, Hunan, 411105, P. R. China. Email address: zhanghanjunxtu@gmail.com
} 
When analyzing absorbing Markov processes, to which the process we discuss in this paper belongs, it is extremely important to study the delay properties, including the quasistationary behavior. In particular, studies have focused on existence as well as uniqueness conditions for quasistationary distributions. For general absorbing continuous-time Markov chains, Ferrari et al. [8] provided an important sufficient condition, usually referred to as the asymptotic remoteness condition, for the existence of quasistationary distributions. See also the very important contribution by Pakes [16], who pointed out that there are many processes that possess a quasistationary distribution without satisfying the asymptotic remoteness condition.

For birth-death processes with killing, quasistationary distributions and related properties have been analyzed by Van Doorn and others. Van Doorn and Zeifman studied the extinction probability and other interesting properties of birth-death processes with killing in [23] and [24], respectively.

Quasistationary distributions for birth-death processes with killing were studied in [6]. Recently, Van Doorn [21] obtained some further interesting conditions for the existence of quasistationary distributions for birth-death processes with killing and also provided some challenging conjectures regarding more general absorbing continuous-time Markov chains with killing. We note that, for one-dimensional diffusion processes with killing, quasistationary distributions were recently investigated by Kolb and Steinsaltz [15]. See also the earlier paper [18]. As early as 1959, Feller [7] showed that a close relationship exists between birth-death processes and one-dimensional diffusion processes, and, thus, that the two processes with killing share similar quasistationary behavior.

In spite of the abovementioned progress, the decay properties of MBPs with disasters have not been fully discussed. To the authors' knowledge, even the most basic problem of determining the exact value of the corresponding decay parameter (see [14]) has not been solved. The main aim of this paper is to fill this gap by considering the decay properties of MBPs with disasters. More specifically, we define the model by specifying the infinitesimal characteristic, i.e. the so-called $q$-matrix, as follows, where $\mathbb{Z}_{+}=\{0,1,2, \ldots\}$ denotes the set of nonnegative integers.

Definition 1.1. A $q$-matrix $Q=\left(q_{i j} ; i, j \in \mathbb{Z}_{+}\right)$is called a branching $q$-matrix with disaster (henceforth referred to as a BWD $q$-matrix) if

$$
q_{i j}= \begin{cases}i\left(b_{j-i+1}-a \delta_{i j}\right) & \text { if } j \geq i-1, i \geq 1 \\ i a & \text { if } j=0 \\ 0 & \text { otherwise }\end{cases}
$$

where $a>0, b_{j} \geq 0, j \neq 1,-b_{1}=\sum_{j \neq 1} b_{j}$, and $\delta_{i j}$ is the Kronecker symbol.

To avoid discussing trivial cases, we assume throughout this paper that

$$
b_{0}>0 \text { and } \sum_{j=2}^{\infty} b_{j}>0 .
$$

We show later that any BWD $q$-matrix is regular and, thus, that there exists only one $Q$-function, the Feller minimal $Q$-function which is honest and satisfies both the Kolmogov backward and forward equations.

Note that, under condition (1.2), the class $\mathcal{C}=\mathbb{Z}_{+} \backslash\{0\}$ is an irreducible class for the $q$-matrix $Q$ as well as for the corresponding Feller minimal $Q$-process. It then follows from, for 
example, [14] that there exists a nonnegative number, denoted by $\lambda_{C}$, called the decay parameter of the corresponding process such that, for all $i, j \in \mathcal{C},(1 / t) \log p_{i j}(t) \rightarrow-\lambda_{C}$ as $t \rightarrow+\infty$.

As is well known, there exists a close link between the important concepts of the decay parameter and the invariant measure, invariant vector, and quasistationary distribution.

An elementary but detailed discussion of the theory regarding decay parameters and quasistationary distributions is given in [1]. For more recent developments, we refer the reader to the good survey paper of Van Doorn and Pollett [22]. For more related works, see [9], [14], [17], [19], [20], and [25], among others.

The structure of this short paper is as follows. In Section 2 we consider the uniqueness, construction, and extinction probability of the MBPs with disaster. In Section 3 we concentrate on studying the decay parameter and the quasistationary distributions. We give the exact value of the decay parameter in explicit form. After showing that our process is always $\lambda_{C}$-positive, we present the corresponding invariant measure and invariant vector. The generating function form for the quasistationary distribution is also given.

\section{Uniqueness, construction, and extinction}

Our known data, the sequence $\left\{b_{j} ; j \geq 0\right\}$ and the constant $a>0$, packaged into a generating function will play an important role in our later analysis. Let $B(s)=\sum_{j=0}^{\infty} b_{j} s^{j}$ and $G(s)=B(s)-a s$. Clearly, these functions have the same convergence radius $\rho=$ $1 / \lim \sup _{n \rightarrow \infty} \sqrt[n]{b_{n}}$. Obviously, $\rho \geq 1$. The properties of $B(s)$ are well known and those of $G(s)$ are very similar. For convenience, in the following lemma we list the useful properties that we use later. The proof is trivial and thus omitted.

Lemma 2.1. (i) The function $G(s)$ has no zero on the complex unit circle $\{z ;|z|=1\}$ and has a unique zero on the complex open disk $\{z ;|z|<1\}$. Furthermore, this unique zero, denoted by $q$, is a positive real number.

(ii) $G(s)$ is a convex function of $s$ on $[0, \rho)$.

(iii) If $\sum_{j=2}^{\infty}(j-1) b_{j} \leq a+b_{0}$ then $G(s)$ is a strictly decreasing function of s on [0,1], while if $\sum_{j=2}^{\infty}(j-1) b_{j}>a+b_{0}$ (including $\left.\sum_{j=2}^{\infty}(j-1) b_{j}=+\infty\right)$ then there exists an $\eta \in(q, 1)$ such that $G(s)$ is strictly decreasing on $[0, \eta)$ and strictly increasing on $[\eta, 1]$.

(iv) At the unique zero $q>0$ of $G(s)$, we have $G^{\prime}(q)<0$.

Our first result is that any BWD $q$-matrix is always regular and, thus, that the corresponding Feller minimal process is honest.

Theorem 2.1. Any BWD q-matrix $Q=\left\{q_{i j}\right\}$ as defined above is regular and, thus, the Feller minimal $Q$-function is honest.

Proof. Let $\Phi(\lambda)=\left\{\phi_{i j}(\lambda) ; i, j \in \mathbb{Z}_{+}\right\}$be the Feller minimal $Q$-resolvent. Then, for each $\lambda>0, \lambda \sum_{j=0}^{\infty} \phi_{i j}(\lambda)$ is the minimal nonnegative solution of the nonnegative linear equation

$$
x_{i}=\sum_{k \neq i} \frac{q_{i k}}{\lambda+q_{i}} x_{k}+\frac{\lambda}{\lambda+q_{i}}, \quad i \in \mathbb{Z}_{+} ;
$$

see, for example, [11] 
Let $x_{i}^{*}(\lambda)=\lambda \sum_{j=0}^{\infty} \phi_{i j}(\lambda), i \geq 0$. Then $x_{0}^{*}(\lambda)=1$ since 0 is an absorbing state and $x_{i}^{*}(\lambda) \in[0,1]$ for $i \geq 1$. Now, by (2.1) we have, for $i \geq 1$,

$$
x_{i}^{*}(\lambda) \geq \frac{q_{i 0}}{\lambda+q_{i}} x_{0}^{*}(\lambda)+\frac{\lambda}{\lambda+q_{i}}=\frac{\lambda+i a}{\lambda+i(a+b)}=1-\frac{b}{\lambda / i+a+b},
$$

where we set $b=-b_{1}>0$. The above inequality together with the fact $x_{0}^{*}(\lambda)=1$ implies that $\inf _{i \geq 0} x_{i}^{*}(\lambda) \geq a /(a+b)>0$. Therefore, (2.1) does not have a nonnegative solution $\left\{y_{i} ; i \geq\right.$ $0\}$ such that $\inf _{i \geq 0} y_{i}=0$. It follows from Theorem 9.2.1 of [11] that $x_{i}^{*}(\lambda) \equiv \lambda \sum_{j=0}^{\infty} \phi_{i j}(\lambda)=$ 1 for all $i \geq 0$. This completes the proof.

Our next aim is to determine the structure of this honest minimal $Q$-function. To this end, we consider the following nonconservative $q$-matrix $Q^{*}=\left\{q_{i j}^{*} ; i, j \geq 0\right\}$ :

$$
q_{i j}^{*}= \begin{cases}i b_{j-i+1}-a \delta_{i j} & \text { if } j \geq i-1, i \geq 1 \\ 0 & \text { otherwise. }\end{cases}
$$

The only difference between $Q$ and $Q^{*}$ are the entries of the first column. Let $P^{*}(t)=$ $\left\{p_{i j}^{*}(t) ; i, j \geq 0\right\}$ be the Feller minimal $Q^{*}$-function. We shall see that the structure of $P^{*}(t)$ is very similar to an ordinary MBP. Define $F_{i}^{*}(t, s)=\sum_{j=0}^{\infty} p_{i j}^{*}(t) s^{j}, i \geq 0$, and $F^{*}(t, s)=$ $F_{1}^{*}(t, s)$. Now, using the Kolmogorov forward equations, we immediately obtain the following basic partial differential equation:

$$
\frac{\partial F_{i}^{*}(t, s)}{\partial t}=G(s) \frac{\partial F_{i}^{*}(t, s)}{\partial s} .
$$

Let $\sigma(t)=\sum_{j=0}^{\infty} p_{1 j}^{*}(t)$ and $\rho(t)=p_{10}^{*}(t)$. Note that $\rho(t)$ defined here has no relation with the $\rho$ defined above as the convergence radius of $G(s)$ and $B(s)$. Using (2.2), we can immediately prove the following lemma.

Lemma 2.2. For the $Q^{*}$-function $P^{*}(t)=\left\{p_{i j}^{*}(t) ; i, j \geq 0\right\}$, we have

(i) $F_{i}^{*}(t, s)=\left(F^{*}(t, s)\right)^{i}, i \geq 0$;

(ii) $\partial F^{*}(t, s) / \partial t=G\left(F^{*}(t, s)\right)$ or, equivalently, $\int_{s}^{F^{*}(s, t)} \mathrm{d} y / G(y)=t$;

(iii) $\mathrm{d} \sigma(t) / \mathrm{d} t=G(\sigma(t))$ or, equivalently, $\int_{1}^{\sigma(t)} \mathrm{d} y / G(y)=t$;

(iv) $\mathrm{d} \rho(t) / \mathrm{d} t=G(\rho(t))$ or, equivalently, $\int_{0}^{\rho(t)} \mathrm{d} y / G(y)=t$.

A direct consequence of Lemma 2.2 is the following corollary.

Corollary 2.1. For the minimal Feller $Q^{*}$-function $P^{*}(t)=\left\{p_{i j}^{*}(t)\right\}$, we have

(i) $\sum_{j=0}^{\infty} p_{i j}^{*}(t)=(\sigma(t))^{i}, i \geq 0$;

(ii) $p_{i 0}^{*}(t)=(\rho(t))^{i}$;

(iii) $\sum_{j=0}^{\infty} p_{k j}^{*}(t) \downarrow q^{k}$ and $p_{k 0}^{*}(t) \uparrow q^{k}$ as $t \rightarrow \infty, k \geq 1$, where $q$ is the unique zero of $G(s)$ given in Lemma 2.1;

(iv) $\lim _{t \rightarrow \infty} \sum_{j=1}^{\infty} p_{k j}^{*}(t)=0$.

Proof. Parts (i) and (ii) are direct consequences of Lemma 2.2(i). Using exactly the same method as in [4], we easily prove (iii). Then (iv) follows directly from (iii). 
The second main result of this section is the construction theorem of the $Q$-function for a BWD $q$-matrix $Q$.

Theorem 2.2. Suppose that $Q$ is a BWD q-matrix as defined in (1.1). Then there exists only one $Q$-function, the Feller minimal $Q$-function, $P(t)=\left\{p_{i j}(t) ; i, j \geq 0\right\}$, whose structure is as follows.

(i) $p_{0 j}(t)=\delta_{0 j}$.

(ii) For all $i \geq 1$ and $j \geq 1, p_{i j}(t)=p_{i j}^{*}(t)$, where $\left\{p_{i j}^{*}(t)\right\}$ is the Fellerminimal $Q^{*}$-function constructed in Lemma 2.2.

(iii) $p_{i 0}(t)=1+(\rho(t))^{i}-(\sigma(t))^{i}, i \geq 1$, where $\rho(t)$ and $\sigma(t)$ are defined above.

Proof. Part (i) follows from the fact that 0 is an absorbing state. Part (ii) is a direct consequence of the resolvent decomposition theorem; see [3] and [4]. It can also easily be proved directly. Part (iii) follows from Theorem 2.1 and Lemma 2.2.

We are now ready to consider the properties of the MBP with disaster. Recall that, for the MBP without disaster, the process may be explosive. However, we shall see that this is not the case if disaster occurs.

Theorem 2.3. For the MBP with disaster, the extinction probability is always 1 , starting from any initial distribution.

Proof. We only need to show that, for all $i \geq 1$, we have $\lim _{t \rightarrow \infty} p_{i 0}(t)=1$. However, this follows directly from Theorem 2.2 and Corollary 2.1.

This result is intuitively quite interesting, though not surprising. It states that some tiny disaster could prevail over a very strong possibility of explosion when disaster does not happen. Even more interesting, we can show that the mean extinction times are finite and even bounded.

To this end, let $\left\{X_{t} ; t \geq 0\right\}$ be the MBP with disaster, let $T=\inf \{t \geq 0 ; X(t)=0\}$ be the extinction time, and let $E_{i}(T)$ be the mean extinction time when the process starts from $i \geq 1$.

Theorem 2.4. For all $i \geq 1, E_{i}(T)<+\infty$. Moreover, we further have $\sup _{i \geq 1} E_{i}(T) \leq 1 / a$.

Proof. Let $y_{i}^{*}=E_{i}(T), i \geq 1$. Then by using, e.g. Theorem 9.4.1 of [11], we know that $\left\{y_{i}^{*} ; i \geq 1\right\}$ is the minimal nonnegative solution of the equation

$$
x_{i}=\frac{1}{q_{i}} \sum_{k \neq i} q_{i k} x_{k}+\frac{1}{q_{i}}, \quad i \geq 1,
$$

with $x_{0}=0$.

If we let $y_{i}=1 / a, i \geq 1$, and $y_{0}=0$ in (2.3), then, for all $i \geq 1$, we obtain

$$
\frac{1}{q_{i}} \sum_{k \neq i} q_{i k} y_{k}+\frac{1}{q_{i}}=\frac{1}{q_{i}} \sum_{k \neq i ; k \geq 1} q_{i k} y_{k}+\frac{1}{q_{i}} \leq \frac{1}{a q_{i}}\left(q_{i}-a\right)+\frac{1}{q_{i}}=\frac{1}{a}=y_{i},
$$

and, thus, by the comparison theorem (see Theorem 3.3.1 of [11]) we obtain $E_{i}(T) \leq 1 / a$ for all $i \geq 1$. 


\section{Decay parameter and quasistationary distributions}

We now consider the decay properties of the MBP with disaster, focusing on the decay parameter and quasistationary distributions.

Recall that the class $\mathcal{C}$ is an irreducible class for the BWD $q$-matrix $Q$ as well as for the Feller minimal $Q$-function. Therefore, the decay parameter $\lambda_{C}$ does exist. To find $\lambda_{C}$, we first show the following result.

Lemma 3.1. There exists a $\mu$-invariant vector of $Q$ on $\mathcal{C}$ for $\mu=(-1) G^{\prime}(q)>0$, with the corresponding $\mu$-invariant vector given by $x_{j}=j q^{j-1}, j \geq 1$, where $q$ is again the unique zero of $G(s)$ on the unit disk $\{z ;|z| \leq 1\}$.

Proof. We only need to show that, for all $i \geq 1$, we have $\sum_{j=1}^{\infty} q_{i j} x_{j}=G^{\prime}(q) x_{i}$. Indeed, for $i=1$, we have $\sum_{j=1}^{\infty} q_{1 j} x_{j}=\left(b_{1}-a\right)+\sum_{j=2}^{\infty} b_{j} j q^{j-1}=G^{\prime}(q)=G^{\prime}(q) x_{1}$. For $i \geq 2$, we obtain

$$
\begin{aligned}
\sum_{j=1}^{\infty} q_{i j} x_{j} & =\sum_{j=i-1}^{\infty} i b_{j-i+1} x_{j}-i a x_{i} \\
& =i\left[\sum_{j=0}^{\infty} b_{j} x_{j+i-1}-a x_{i}\right] \\
& =i\left[\sum_{j=0}^{\infty} b_{j}(j+i-1) q^{j+i-2}-a i q^{i-1}\right] \\
& =i q^{i-1}\left[\sum_{j=1}^{\infty} j b_{j} q^{j-1}-a+\frac{i-1}{q}\left(\sum_{j=0}^{\infty} b_{j} q^{j}-a q\right)\right] \\
& =i q^{i-1}\left[G^{\prime}(q)+\frac{i-1}{q} G(q)\right] \\
& =i q^{i-1} G^{\prime}(q) \\
& =G^{\prime}(q) x_{i} .
\end{aligned}
$$

This completes the proof.

We can now present our first important conclusion regarding the decay parameter.

Theorem 3.1. For the MBP with disaster, the decay parameter $\lambda_{C}$ is given by $\lambda_{C}=$ $(-1) G^{\prime}(q)>0$.

Proof. It follows from Lemma 3.1 that $\lambda_{C} \geq(-1) G^{\prime}(q)$; hence, we only need to show that $\lambda_{C} \leq(-1) G^{\prime}(q)$. Assume that this is not true. Then there exists a $\mu>0$ such that $(-1) G^{\prime}(q)<\mu<\lambda_{C}$. Since $\mu<\lambda_{C}$, we know that, for all $i, j \geq 1, \int_{0}^{\infty} \mathrm{e}^{\mu t} p_{i j}(t) \mathrm{d} t<\infty$, which, using Theorem 2.2(ii), is just

$$
\int_{0}^{\infty} \mathrm{e}^{\mu t} p_{i j}^{*}(t) \mathrm{d} t<\infty, \quad i, j \geq 1 .
$$

However, by the Kolmogorov forward equation for $Q^{*}$, i.e. $\mathrm{d} P^{*}(t) / \mathrm{d} t=P^{*}(t) Q$, we know that $\mathrm{d} p_{i 0}^{*}(t) / \mathrm{d} t=b_{0} p_{i 1}^{*}(t)$ and, thus, (3.1) implies that (recall that $b_{0}>0$ )

$$
\int_{0}^{\infty} \mathrm{e}^{\mu t} \frac{\mathrm{d} p_{i 0}^{*}(t)}{\mathrm{d} t} \mathrm{~d} t<\infty .
$$


We now show that (3.2) implies that

$$
\lim _{t \rightarrow \infty} \mathrm{e}^{\mu t}\left(q^{i}-p_{i 0}^{*}(t)\right)=0
$$

and

$$
\int_{0}^{\infty} \mathrm{e}^{\mu t}\left(q^{i}-p_{i 0}^{*}(t)\right) \mathrm{d} t<\infty .
$$

Of course, (3.3) is an immediate consequence of (3.4), but we need to show (3.3) first. To claim (3.3), we use the fact that $p_{i 0}^{*}(t) \uparrow q^{i}$ as $t \rightarrow \infty$; see Corollary 2.1. Thus,

$$
0 \leq \mathrm{e}^{\mu t}\left(q^{i}-p_{i 0}^{*}(t)\right)=\mathrm{e}^{\mu t} \int_{t}^{\infty} \frac{\mathrm{d} p_{i 0}^{*}(s)}{\mathrm{d} s} \mathrm{~d} s .
$$

This implies that

$$
0 \leq \mathrm{e}^{\mu t}\left(q^{i}-p_{i 0}^{*}(t)\right) \leq \int_{t}^{\infty} \mathrm{e}^{\mu s} \frac{\mathrm{d} p_{i 0}^{*}(s)}{\mathrm{d} s} \mathrm{~d} s .
$$

Letting $t \rightarrow \infty$ in (3.5) and applying (3.2) immediately yields (3.3). We now use (3.3) to show that (3.4) is also true. Indeed, by integration by parts and noting that $(-1) G^{\prime}(q)<\mu<\lambda_{C}$, we obtain, for any constant $T$ such that $0<T<\infty$,

$$
\int_{0}^{T} \mathrm{e}^{\mu t} \frac{\mathrm{d} p_{i 0}^{*}(t)}{\mathrm{d} t} \mathrm{~d} t=q^{i}-\mathrm{e}^{\mu T}\left(q^{i}-p_{i 0}^{*}(T)\right)+\mu \int_{0}^{T} \mathrm{e}^{\mu t}\left(q^{i}-p_{i 0}^{*}(t)\right) \mathrm{d} t .
$$

Letting $T \rightarrow+\infty$ in the above equality, and applying (3.2) and (3.3), we obtain

$$
+\infty>\int_{0}^{\infty} \mathrm{e}^{\mu t} \frac{\mathrm{d} p_{i 0}^{*}(t)}{\mathrm{d} t} \mathrm{~d} t=q^{i}+\mu \int_{0}^{\infty} \mathrm{e}^{\mu t}\left(q_{i}-p_{i 0}^{*}(t)\right) \mathrm{d} t,
$$

which shows that (3.4) is true. However, $q^{i}$ is the extinction probability of the $Q^{*}$-process when the process starts from state $i$. It therefore follows that $\sum_{j=0}^{\infty} p_{i j}^{*}(t) q^{j}=q^{i}$ for all $i \geq 1$, and so (3.4) can be rewritten as

$$
\int_{0}^{\infty} \mathrm{e}^{\mu t}\left(\sum_{j=1}^{\infty} p_{i j}^{*}(t) q^{j}\right) \mathrm{d} t<+\infty
$$

We now rewrite (2.2) as

$$
\frac{\partial}{\partial t} \sum_{j=0}^{\infty} p_{i j}^{*}(t) s^{j}=G(s) \sum_{j=1}^{\infty} j p_{i j}^{*}(t) s^{j-1} .
$$

Multiplying both sides of this equality by $\mathrm{e}^{\mu t}$ yields

$$
\mathrm{e}^{\mu t} \frac{\mathrm{d} p_{i 0}^{*}(t)}{\mathrm{d} t}+\sum_{k=1}^{\infty} \mathrm{e}^{\mu t} \frac{\mathrm{d} p_{i k}^{*}(t)}{\mathrm{d} t} s^{k}=G(s) \sum_{k=1}^{\infty} k \mathrm{e}^{\mu t} p_{i k}^{*}(t) s^{k-1} .
$$

Integrating (3.7) from 0 to $\infty$ yields, for $0 \leq s<q$,

$$
\begin{gathered}
\int_{0}^{\infty} \mathrm{e}^{\mu t} \frac{\mathrm{d} p_{i 0}^{*}(t)}{\mathrm{d} t} \mathrm{~d} t+\sum_{k=1}^{\infty}\left(\int_{0}^{\infty} \mathrm{e}^{\mu t} \frac{\mathrm{d} p_{i k}^{*}(t)}{\mathrm{d} t} \mathrm{~d} t\right) s^{k} \\
=G(s) \sum_{k=1}^{\infty} k\left(\int_{0}^{\infty} \mathrm{e}^{\mu t} p_{i k}^{*}(t) \mathrm{d} t\right) s^{k-1} .
\end{gathered}
$$


Note that all the three integrals in (3.8) are finite and, thus, (3.8) makes sense. Indeed, (3.6) together with the fact that $G(s)>0$ for $0 \leq s<q$ guarantees that the right-hand side of (3.8) is finite for $0 \leq s<q$, while (3.2) guarantees that the first term on the left-hand side is finite. These two facts then imply that the second term on the left-hand side is also finite since all three terms are nonnegative.

Note that (3.1) also implies that $\lim _{t \rightarrow \infty} \mathrm{e}^{\mu t} p_{i k}^{*}(t)=0$ for all $i, k \geq 1$ and, hence, (3.8) can be rewritten as

$$
\int_{0}^{\infty} \mathrm{e}^{\mu t} \frac{\mathrm{d} p_{i 0}^{*}(t)}{\mathrm{d} t} \mathrm{~d} t-s^{i}=\mu \sum_{k=1}^{\infty}\left(\int_{0}^{\infty} \mathrm{e}^{\mu t} p_{i k}^{*}(t) \mathrm{d} t\right) s^{k}+G(s) \sum_{k=1}^{\infty} k\left(\int_{0}^{\infty} \mathrm{e}^{\mu t} p_{i k}^{*}(t) \mathrm{d} t\right) s^{k-1}
$$

for all $i \geq 1$ and $0 \leq s<q$. Differentiating with respect to $s$ over the interval $[0, q)$ yields

$$
\begin{aligned}
(-i) s^{i-1}= & \left(\mu+G^{\prime}(s)\right) \sum_{k=1}^{\infty}\left(\int_{0}^{\infty} \mathrm{e}^{\mu t} p_{i k}^{*}(t) \mathrm{d} t\right) k s^{k-1} \\
& +G(s) \sum_{k=1}^{\infty} k(k-1)\left(\int_{0}^{\infty} \mathrm{e}^{\mu t} p_{i k}^{*}(t) \mathrm{d} t\right) s^{k-2} .
\end{aligned}
$$

Note that $0<q<1$ and that all the terms in the above equality are finite for $s=q$. Thus, the above equality actually holds for $s=q$. More exactly, by letting $s \uparrow q$ in the above equality, we easily obtain

$$
(-i) q^{i-1}=\left(\mu+G^{\prime}(q)\right) \sum_{k=1}^{\infty}\left(\int_{0}^{\infty} \mathrm{e}^{\mu t} p_{i k}^{*}(t) \mathrm{d} t\right) k q^{k-1} .
$$

Since the second multiplier on the right-hand side of (3.9) is a positive finite number, $\mu<$ $(-1) G^{\prime}(q)$, which contradicts $(-1) G^{\prime}(q)<\mu<\lambda_{C}$. Hence, $\lambda_{C} \leq(-1) G^{\prime}(q)$, which completes the proof.

Using the known sequence $\left\{b_{j} ; j \geq 0\right\}$ and the parameter $a>0$, the decay parameter $\lambda_{C}=(-1) G^{\prime}(q)$ can be rewritten as $\lambda_{C}=a+b_{0}+\sum_{j=2}^{\infty} b_{j}-\sum_{j=2}^{\infty} j b_{j} q^{j-1}$, where $q$ is the unique zero of $G(s)$. As the exact value of $q$ is in many cases quite difficult to obtain, we instead provide some bounds that can be easily calculated. We give lower and upper bounds in the following two corollaries whose proofs are straightforward and are thus omitted.

Corollary 3.1. Let $\lambda_{C}$ be the decay parameter given in Theorem 3.1.

(i) If $\sum_{j=2}^{\infty}(j-1) b_{j} \leq b_{0}$ then $\lambda_{C}>a+b_{0}-\sum_{j=2}^{\infty}(j-1) b_{j} \geq a$.

(ii) If $\sum_{j=2}^{\infty}(j-1) b_{j}>b_{0}$ (including the case $\left.\sum_{j=2}^{\infty}(j-1) b_{j}=+\infty\right)$ then

$$
\lambda_{C}>a+b_{0}+\sum_{j=2}^{\infty} b_{j}-\sum_{j=2}^{\infty} j b_{j} \tau^{j-1}>a,
$$

where $0<\tau<1$ is the unique zero of $B(s)$ on $(0,1)$.

In particular, for both cases, we have $\lambda_{C}>a$. 
Corollary 3.2. The decay parameter $\lambda_{C}$ satisfies

$$
\lambda_{C}<a+b-\sum_{j=2}^{\infty} j b_{j}\left(\frac{b_{0}}{a+b}\right)^{j-1} .
$$

Moreover, if $b_{2}>0$ then $\lambda_{C} \leq a+b-\sum_{j=2}^{\infty} j b_{j} q_{c}^{j-1}$, where $b=-b_{1}$ and $q_{c}=$ $\left((a+b)-\sqrt{\left.(a+b)^{2}-4 b_{0} b_{2}\right)} / 2 b_{2}\right.$. Furthermore, the inequality above becomes an equality if and only if $b_{k}=0$ for all $k \geq 3$.

The lower and upper bounds obtained in Corollaries 3.1 and 3.2 are meaningful since in many situations it is quite easy to find the smallest nonnegative zero of $B(s)$ but quite difficult to find the smallest zero of $G(s)$.

Upon obtaining the decay parameter $\lambda_{C}$, we now determine whether or not the process is $\lambda_{C}$-recurrent, which is straightforward.

Theorem 3.2. The MBP with disaster is $\lambda_{C}$-recurrent.

Proof. Suppose that it is $\lambda_{C}$-transient. Then $\int_{0}^{\infty} \mathrm{e}^{\lambda_{C} t} p_{i j}(t) \mathrm{d} t<\infty$ for all $i, j \geq 1$. Under these conditions, we can easily see that the proof of Theorem 3.1 applies and, thus, we again obtain

$$
(-i) q^{i-1}=\left(\lambda_{C}+G^{\prime}(q)\right) \sum_{k=1}^{\infty}\left(\int_{0}^{\infty} \mathrm{e}^{\lambda_{c} t} p_{i k}(t) \mathrm{d} t\right) k q^{k-1}, \quad i \geq 1 .
$$

Then, as before, we obtain $\lambda_{C}<(-1) G^{\prime}(q)$, which contradicts Theorem 3.1.

In order to investigate the property of $\lambda_{C}$-positive recurrence further, we need to obtain the $\lambda_{C}$-invariant measure. Since we have just proved that the MBP with disaster is $\lambda_{C}$-recurrent, then it follows from [14], or Theorem 5.2.8 of [1, p. 177], that there exists a unique (in the sense of up to constant multiples) $\lambda_{C}$-invariant measure as well as a unique (up to constant multiples) $\lambda_{C}$-invariant vector. The unique $\lambda_{C}$-invariant vector was obtained in Lemma 3.1. We now investigate the unique $\lambda_{C}$-invariant measure. In the following theorem we give the generating function of the unique $\lambda_{C}$-invariant measure $\left\{m_{j} ; j \geq 1\right\}$. Since the uniqueness of the $\lambda_{C}$-invariant measure is in the sense of up to constant multiples, we assume without loss of generality that $m_{1}=1$.

Theorem 3.3. For the MBP with disaster, there exists a unique (up to constant multiples) $\lambda_{C^{-}}$ invariant measure $\left\{m_{j} ; j \geq 1\right\}$. The generating function of this $\lambda_{C}$-invariant measure (with $m_{1}=1$ ) is given for $-q<s<q$ by

$$
M(s)=\sum_{j=1}^{\infty} m_{j} s^{j}=\int_{0}^{s} \exp \left\{-\int_{0}^{t} \frac{G^{\prime}(x)-G^{\prime}(q)}{G(x)} \mathrm{d} x\right\} \mathrm{d} t,
$$

where $q$ is again the unique positive zero of $G(s)$ on the complex unit disk $\{z ; z \leq 1\}$.

Proof. The existence and uniqueness of the $\lambda_{C}$-invariant measure $\left\{m_{j} ; j \geq 1\right\}$ follow from Theorem 3.2 and Kingman's lemma (see [14]). Then this invariant measure is also the $\lambda_{C^{-}}$ invariant measure of the BWD $q$-matrix $Q$ on $\mathcal{C}=\{1,2, \ldots\}$. Hence, we have

$$
\sum_{k=1}^{\infty} m_{k} q_{k j}=-\mu m_{j}, \quad j \geq 1,
$$


or, by noting the structure of the BWD $q$-matrix $Q$,

$$
\sum_{k=1}^{j+1} m_{k} k b_{j-k+1}+m_{j}(-j a)=-\mu m_{j}, \quad j \geq 1,
$$

where $\mu=(-1) G^{\prime}(q)$ denotes the decay parameter.

By (3.11), it is easily seen that, similarly as proved in [5] (see the proof of Theorem 5.1 therein), the generating function of this unique $\lambda_{C}$-invariant measure $M(s)=\sum_{j=1}^{\infty} m_{j} s^{j}$ is convergent at least in a sufficiently small interval $(-\tau, \tau)$. Multiplying $s^{j}$ on (3.11) and summing $j$ from 1 to $\infty$ for $s \in(-\tau, \tau)$ immediately yields

$$
\sum_{j=1}^{\infty}\left(\sum_{k=1}^{j+1} m_{k} k b_{j-k+1}\right) s^{j}-a \sum_{j=1}^{\infty} j m_{j} s^{j}=-\mu \sum_{j=1}^{\infty} m_{j} s^{j} .
$$

Applying Fubini's theorem to the first term on the left-hand side of the above equality together with a trivial algebra yields

$$
\left(\sum_{j=0}^{\infty} b_{j} s^{j}-a s\right) \sum_{k=1}^{\infty} k m_{k} s_{k-1}+\mu \sum_{k=1}^{\infty} m_{k} s^{k}=m_{1} b_{0},
$$

i.e.

$$
G(s) M^{\prime}(s)+\mu M(s)=m_{1} b_{0} .
$$

By noting that $G(s)$ in (3.12) has only one zero, $q$, in the complex unit disk $\{z ;|z| \leq 1\}$, we know that $M(s)$ is at least well defined on the complex disk $\{z ;|z|<q\}$. Consequently, both $G(s)$ and $M(s)$ are infinitely differentiable on the disk $\{z ;|z|<q\}$. Now differentiating (3.12) with respect to $s$ within the interval $(-q, q)$ yields

$$
\left(G^{\prime}(s)+\mu\right) M^{\prime}(s)+G(s) M^{\prime \prime}(s)=0 .
$$

However, both $M^{\prime}(s)$ and $G(s)$ are strictly positive on the interval $[0, q)$ and, thus, (3.13) can be rewritten as

$$
\frac{M^{\prime \prime}(s)}{M^{\prime}(s)}=-\frac{G^{\prime}(s)+\mu}{G(s)} .
$$

Noting that $m_{1}=1$ and, thus, $\ln M^{\prime}(0)=0$, using (3.14) we obtain

$$
M^{\prime}(t)=c \exp \left\{-\int_{0}^{t} \frac{G^{\prime}(s)+\mu}{G(s)} \mathrm{d} s\right\}, \quad 0 \leq t<q .
$$

By noting that $M^{\prime}(0)=1$, once again, we know that the constant $c$ in the above is equal to 1 . Hence, by noting that $\mu=(-1) G^{\prime}(q)=\lambda_{C}$, we obtain

$$
M^{\prime}(t)=\exp \left\{-\int_{0}^{t} \frac{G^{\prime}(s)-G^{\prime}(q)}{G(s)} \mathrm{d} s\right\}, \quad 0 \leq t<q .
$$

Now (3.10) follows from (3.15) directly by noting $M(0)=0$. The proof is complete.

In fact, we can do even better. Indeed, the following corollary shows that the convergence radius of $M(s)$ in (3.10) can be further expanded to the complex unit disk $\{z ;|z| \leq 1\}$. Although this is an easy corollary, it has significant consequences for the quasistationary distribution of the MBP with disaster. 
Corollary 3.3. Let $M(s)=\sum_{j=1}^{\infty} m_{j} s^{j}$ be the generating function of the $\lambda_{C}$-invariant measure for the $M B P$ with disaster. Then, for any $-1<s<1$, we have $M(s)<+\infty$ and $M^{\prime}(s)<+\infty$. In particular, we have $M^{\prime}(q)<+\infty$.

Proof. Following from Theorem 3.3 we know that (3.10) holds for any $0 \leq s<q$. Now let $s \uparrow q$ in (3.10). Note that $q$ is the single zero of $G(s)$ and, thus,

$$
\lim _{s \uparrow q} \frac{G^{\prime}(s)-G^{\prime}(q)}{G(s)}=\frac{G^{\prime \prime}(q)}{G^{\prime}(q)},
$$

which is a finite number. Indeed, we know that $G(s)$, as a power series, is convergent for all $|s| \leq 1$, and, hence, both $G^{\prime}(q)$ and $G^{\prime \prime}(q)$ are finite and also $G^{\prime}(q) \neq 0$ since $0<q<1$ is the single zero of $G(s)$. Therefore, the integral $\int_{0}^{q}\left(\left(G^{\prime}(x)-G^{\prime}(q)\right) / G(x)\right) \mathrm{d} x$ is finite. Then it follows from the fact that $G(s)$ has only one zero on $\{z ;|z| \leq 1\}$ and (3.10) that $M(s)<+\infty$ for all $|s| \leq 1$. We also have $M^{\prime}(s)<+\infty$ for all $|s|<1$.

We are now able to show that the MBP with disaster is $\lambda_{C}$-positive-recurrent.

Theorem 3.4. The MBP with disaster is $\lambda_{C}$-positive-recurrent. Moreover, the generating function of the quasistationary distribution $\left\{\pi_{i} ; i \geq 1\right\}$, denoted by $\Pi(s)=\sum_{k=1}^{\infty} \pi_{k} s^{k}$, is given by

$$
\Pi(s)=\frac{\int_{0}^{s} \exp \left\{-\int_{0}^{t}\left(\left(G^{\prime}(x)-G^{\prime}(q)\right) / G(x)\right) \mathrm{d} x\right\} \mathrm{d} t}{\int_{0}^{1} \exp \left\{-\int_{0}^{t}\left(\left(G^{\prime}(x)-G^{\prime}(q)\right) / G(x)\right) \mathrm{d} x\right\} \mathrm{d} t} .
$$

Proof. By Kingman's theorem (see [14] or Theorem 5.2.8 of [1]) we know that the class $\mathcal{C}=\mathbb{Z}_{+} \backslash\{0\}$ is $\lambda_{C}$-positive if and only if $\sum_{k \in \mathcal{C}} m_{k} x_{k}<+\infty$, where $\left\{m_{k} ; k \in \mathcal{C}\right\}$ is a $\lambda_{C}$-invariant measure and $\left\{x_{k} ; k \in \mathcal{C}\right\}$ is a $\lambda_{C}$-invariant vector. However, as shown in Theorem 3.1 and Theorem 3.3, we know that the $\lambda_{C}$-invariant vector and $\lambda_{C}$-invariant measure are respectively given by $x_{j}=j q_{j-1}$ for $j \geq 1$ and (3.10). It then follows from Theorem 3.3 that

$$
\sum_{k \in \mathcal{C}} m_{k} x_{k}=\sum_{k=1}^{\infty} m_{k} k q^{k-1}=M^{\prime}(q)<+\infty,
$$

and, thus, the MBP with disaster is $\lambda_{C}$-positive. Also, by Corollary 3.3 we know that $\sum_{k=1}^{\infty} m_{k}<$ $+\infty$. Hence, the unique quasistationary distribution $\left\{\pi_{i} ; i \geq 1\right\}$ does exist and is given by $\pi_{j}=m_{j} / \sum_{j=1}^{\infty} m_{j}, j \geq 1$. Therefore, the generating function of $\left\{\pi_{j} ; j \geq 1\right\}$, denoted by $\Pi(s)=\sum_{j=1}^{\infty} \pi_{j} s^{j}$, can be written as $\Pi(s)=M(s) / M(1)$. Now, using (3.10), we obtain (3.16), which completes the proof.

\section{Acknowledgements}

The authors would like to thank the anonymous referees for providing very helpful comments and suggestions, which resulted in a much improved version of this paper. Zhang Hanjun acknowledges the support of the Xiangtan University new staff Research Start-up Grant, number 08QDZ27. K. W. Ng acknowledges the financial support of the Endowed Fund of the Patrick S. C. Poon Professorship in Statistics and Actuarial Sciencce at HKU during this project.

\section{References}

[1] Anderson, W. J. (1991). Continuous-Time Markov Chains. An Applications-Oriented Approach. Springer, New York.

[2] Athreya, K. B. And Ney, P. E. (1972). Branching Processes. Springer, New York. 
[3] Chen, A. Y. and Renshaw, E. (1990). Markov branching processes with instantaneous immigration. Prob. Theory Relat. Fields 87, 209-240.

[4] Chen, A. Y. and Renshaw, E. (1993). Existence and uniqueness criteria for conservative uni-instantaneous denumerable Markov processes. Prob. Theory Relat. Fields 94, 427-456.

[5] Chen, A., Li, J., Hou, Z. And NG, K. W. (2010). Decay properties and quasi-stationary distributions for stopped Markovian bulk-arrival and bulk-service queues. Queueing Systems 66, 275-311.

[6] CoOlen-Schrijner, P. ANd VAn Doorn, E. A. (2006). Quasi-stationary distributions for birth-death processes with killing. J. Appl. Math. Stoch. Anal. 2006, $15 \mathrm{pp}$.

[7] Feller, W. (1959). The birth and death processes as diffusion processes. J. Math. Pures Appl. 38, 301-345.

[8] Ferrari, P. A., Kesten, H., Martinez, S. and Picco, P. (1995). Existence of quasi-stationary distributions. A renewal dynamical approach. Ann. Prob. 23, 501-521.

[9] Flaspohler, D. C. (1974). Quasi-stationary distributions for absorbing continuous-time denumerable Markov chains. Ann. Inst. Statist. Math. 26, 351-356.

[10] Harris, T. E. (1963). The Theory of Branching Processes. Springer, Berlin.

[11] Hou, Z. T. And Guo, Q. F. (1988). Homogeneous Denumerable Markov Processes. Springer, Berlin.

[12] Kaplan, N., Sudbury, A. And Nilsen, T. S. (1975). A branching process with disasters. J. Appl. Prob. 12, 47-59.

[13] Karlin, S. and Tavaré, S. (1982). Linear birth and death processes with killing. J. Appl. Prob. 19, $477-487$.

[14] Kingman, J. F. C. (1963). The exponential decay of Markov transition probabilities. Proc. London Math. Soc. (3) 13, 337-358.

[15] Kolb, M. and Steinsaltz, D. (2012). Quasilimiting behavior for one-dimensional diffusions with killing. Ann. Prob. 40, 162-212.

[16] PAKes, A. G. (1995). Quasi-stationary laws for Markov processes: examples of an always proximate absorbing state. Adv. Appl. Prob. 27, 120-145.

[17] Pollett, P. K. (1988). Reversibility, invariance and $\mu$-invariance. Adv. Appl. Prob. 20, 600-621.

[18] Steinsaltz, D. And Evans, S. N. (2007). Quasistationary distributions for one-dimensional diffusions with killing. Trans. Amer. Math. Soc. 359, 1285-1324.

[19] VAN Doorn, E. A. (1985). Conditions for exponential ergodicity and bounds for the decay parameter of a birth-death process. Adv. Appl. Prob. 17, 514-530.

[20] VAN Doorn, E. A. (1991). Quasi-stationary distributions and convergence to quasi-stationarity of birth-death processes. Adv. Appl. Prob. 23, 683-700.

[21] VAN Doorn, E. A. (2012). Conditions for the existence of quasi-stationary distributions for birth-death processes with killing. Stoch. Process. Appl. 122, 2400-2410.

[22] Van Doorn, E. A. And Pollett, P. K. (2011). Quasi-stationary distributions. Memorandum 1945, Department of Applied Mathematics, University of Twente, Enschede. Available at http://eprints.eemcs.utwente.n1/20245.

[23] Van Doorn, E. A. And Zeifman, A. I. (2005). Birth-death processes with killing. Statist. Prob. Lett. 72, 33-42.

[24] Van Doorn, E. A. and Zeifman, A. I. (2005). Extinction probability in a birth-death process with killing. J. Appl. Prob. 42, 185-198.

[25] Vere-Jones, D. (1962). Geometric ergodicity in denumerable Markov chains. Quart. J. Math. Oxford (2) 13 , $7-28$. 